\title{
Statins Do Not Directly Inhibit the Activity of Major Epigenetic Modifying Enzymes
}

\author{
Stephanie Bridgeman ${ }^{1}{ }^{\circledR}$, Wendy Northrop ${ }^{1}$, Gaewyn Ellison ${ }^{1}{ }^{\circledR}$, Thiru Sabapathy ${ }^{1}$, \\ Phillip E. Melton ${ }^{1,2}$, , Philip Newsholme ${ }^{1}$ and Cyril D. S. Mamotte ${ }^{1, *}$ \\ 1 School of Pharmacy and Biomedical Sciences, and Curtin Health Innovation Research Institute, \\ Curtin University, Bentley, WA 6102, Australia; stephanie.allen@postgrad.curtin.edu.au (S.B.); \\ wendy.northrop@student.curtin.edu.au (W.N.); gaewyn.ellison@postgrad.curtin.edu.au (G.E.); \\ t.sabapathy@postgrad.curtin.edu.au (T.S.); phillip.melton@curtin.edu.au (P.E.M.); \\ Philip.Newsholme@curtin.edu.au (P.N.) \\ 2 Centre for Genetic Origins of Health and Disease, Faculty of Health and Medical Science, the University \\ of Western Australia, Perth, WA 6000, Australia \\ * Correspondence: c.mamotte@curtin.edu.au
}

Received: 28 February 2019; Accepted: 4 April 2019; Published: 10 April 2019

\begin{abstract}
The potential anticancer effects of statins-a widely used class of cholesterol lowering drugs-has generated significant interest, as has the use of epigenetic modifying drugs such as HDAC and DNMT inhibitors. We set out to investigate the effect of statin drugs on epigenetic modifications in multiple cell lines, including hepatocellular carcinoma, breast carcinoma, leukemic macrophages, cervical adenocarcinoma, and insulin-secreting cells, as well as liver extracts from statin-treated C57B1/6J mice. Cells or cell extracts were treated with statins and with established epigenetic modulators, and HDAC, HAT, and DNMT activities were quantified. We also examined histone acetylation by immunoblotting. Statins altered neither HDAC nor HAT activity. Accordingly, acetylation of histones $\mathrm{H} 3$ and $\mathrm{H} 4$ was unchanged with statin treatment. However, statins tended to increase DNMT activity. These results indicate that direct inhibition of the major classes of epigenetic modifying enzymes, as previously reported elsewhere, is unlikely to contribute to any anticancer effects of statins. This study concerned global effects on epigenetic enzyme activities and histone acetylation; whether statins influence epigenetic modifications in certain genomic regions, cannot be ruled out and remains to be investigated.
\end{abstract}

Keywords: HMG-CoA reductase inhibitors; epigenetics; histone acetylation; DNA methylation; HDAC inhibitors; DNMT inhibitors

\section{Introduction}

HMG-CoA reductase inhibitors, commonly known as statins, are widely used to reduce low-density lipoprotein (LDL) cholesterol, with an estimated $>30$ million statin users in the United States alone [1]. By inhibiting HMG-CoA reductase, the key rate-determining step in the mevalonate pathway of cholesterol biosynthesis, statins increase the uptake of LDL from the bloodstream into cells, primarily hepatocytes, thus lowering plasma LDL. Multiple large-scale clinical trials have established the efficacy of statins in reducing morbidity and mortality from cardiovascular disease (CVD). A meta-analysis of 59 placebo-controlled trials encompassing 157,217 participants found statins reduced major coronary events by $31 \%$ and overall mortality by $13 \%$ [2]. In addition to lipid-lowering effects, statins have also been reported to have beneficial pleiotropic effects, including anti-inflammatory, anti-proliferative, and immunomodulatory activities, which may protect against cancer [3]. Meta-analyses of cohort and case-control studies found statin use is associated with a 
reduced risk of multiple types of cancers, including liver, colorectal, gastric, ovarian, hematological, and esophageal cancers [4], although no such association was found in a meta-analysis restricted to randomized controlled trials [5]. Furthermore, a meta-analysis of 95 cohort studies found that amongst cancer patients, statin use decreased all-cause and cancer-specific mortality, including amongst prostate, colorectal, and breast cancer patients [6]. In a recent review, we described the evidence that statins have epigenetic effects which may contribute to anticancer activity [7].

In recent years, there has been a plethora of research on epigenetic modifications and their association with cancer and other common disorders such as CVD and diabetes [8-10]. Epigenetic modifications are changes to DNA that may alter gene expression without altering the base nucleotide code. The two major classes of epigenetic modifications are histone modifications, of which the most studied and best understood is histone acetylation, and DNA methylation. These modifications are carried out by epigenetic modifying enzymes. Histone acetylation, generally associated with increased gene expression, is controlled by two opposing classes of enzymes: histone acetyltransferases (HATs) and histone deacetylases (HDACs). DNA methyltransferases (DNMTs) transfer methyl groups from S-adenosyl methionine to nucleotides, primarily cytosines in CpG dinucleotides, in a process usually associated with transcriptional silencing. There are several reports of statins inhibiting HDAC enzyme activity [11-13] and a single report of DNMT inhibition [14], with the majority of these studies linking such effects to anticancer activity. However, notable gaps remain in the literature. For example, previous reports of an inhibitory effect on HDACs were based on direct enzymatic inhibition of recombinant HDACs or on nuclear extracts, and findings on histone acetylation have been mixed [12,13]. Importantly, the effect of statins on HDAC activity in live cells has not been reported, despite being more relevant to in vivo and treatment models, nor has the effect of statins on HAT activity.

We therefore conducted a comprehensive investigation of the effects of statins on HDAC activity in both live cells and nuclear extracts, using a variety of different cell lines, including HepG2 hepatocellular carcinoma cells, MDA-MB-231 breast carcinoma cells, and HeLa cervical adenocarcinoma extracts. Since statin use has also been associated with diabetes [15], we also used BRIN-BD11 cells, a model of insulin-secreting rat pancreatic $\beta$-cells, and the livers of statin-treated mice, both on a control diet and on a high-fat diet. By using a variety of different cell types, we consider our findings are generally applicable, and there is little evidence to suggest that enzymatic properties of HDACs differ between different cell types. By applying statins directly to cell extracts we could determine if statins inhibit activity through direct interaction with the enzyme, as previously reported for HDAC activity by Lin et al. [12]. On the other hand, by measuring enzymatic activity in treated cells, as previously reported with DNMT activity by Kodach et al. [14], we could determine if statins alter activity either directly or through altered expression of enzymes or other indirect phenotypic changes. For example, the established HDAC inhibitor trichostatin A (TSA) directly inhibits HDAC enzymes through interaction with the active site of HDAC enzymes [16], while the antidiabetic drug metformin is thought to reduce HDAC activity not through direct inhibition, but indirectly through activation of AMP kinase, which subsequently phosphorylates HDAC proteins and thus reduces their enzymatic activity [17]. We also investigated effects on global histone acetylation in treated cells and HAT and DNMT activity in cell extracts.

\section{Results}

\subsection{The Effect of Statins on Cell Viability and Cholesterol}

Dose-response curves for viability and measurement of cellular cholesterol reduction were first conducted to ensure use of appropriate statin doses for later epigenetic studies in live cells. Statins decreased HepG2 cell viability, as assessed by the alamarBlue ${ }^{\circledR}$ assay, in a dose-dependent manner (Figure 1a). This was most apparent for the lipophilic atorvastatin and simvastatin, reducing viability to $13 \%$ ( $\pm 5 \% \mathrm{SEM})$ and 3\% ( $\pm 5 \% \mathrm{SEM})$, respectively, at $500 \mu \mathrm{M}$ after $24 \mathrm{~h}$. The hydrophilic 
pravastatin and rosuvastatin were less toxic, with viability remaining at 70\% ( $\pm 6 \% \mathrm{SEM}$ ) and $83 \%$ $( \pm 17 \%$ SEM), respectively, at $500 \mu \mathrm{M}$. Simvastatin reduced viability at all tested concentrations, however no other statin affected viability at $10 \mu \mathrm{M}$ in HepG2 cells, and no statin affected viability in MDA-MB-231 breast carcinoma cells at $10 \mu \mathrm{M}$ (Figure S1). Furthermore, all statins significantly lowered HepG2 cellular cholesterol at $10 \mu \mathrm{M}$ after $24 \mathrm{~h}$ treatment (Figure $1 \mathrm{~b})$, ranging from $33 \%( \pm 14 \%$ SEM) lower cholesterol by pravastatin to $53 \%$ ( \pm 8 SEM) by simvastatin, demonstrating that $10 \mu \mathrm{M}$ is a sufficient concentration for HMG-CoA reductase inhibition by statins. As such this concentration was used for subsequent experiments.

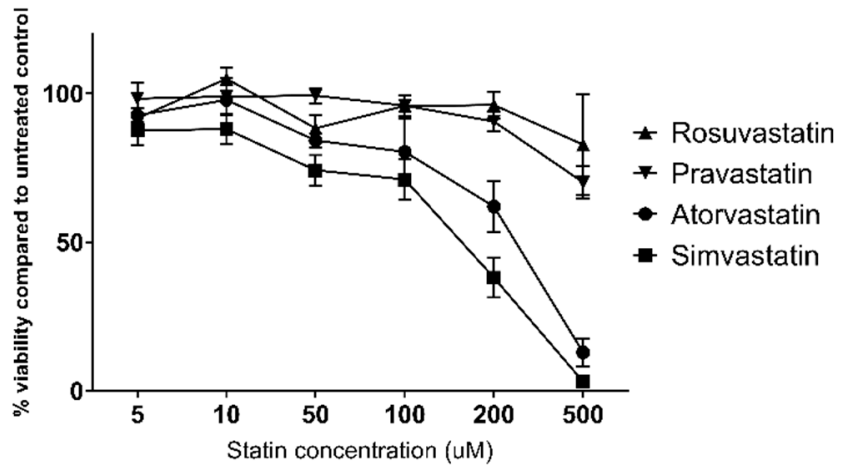

(a)

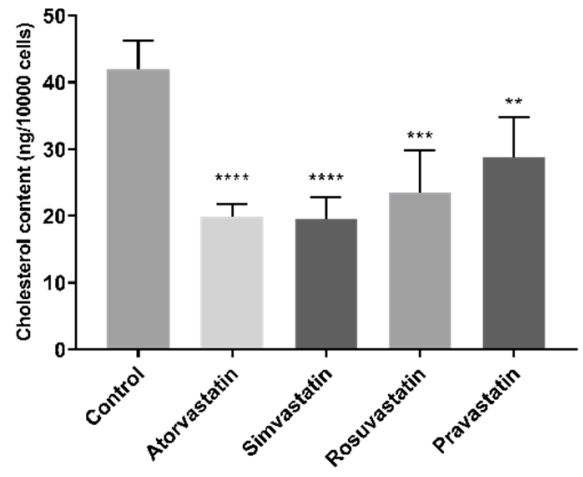

(b)

Figure 1. (a) HepG2 cell viability following $24 \mathrm{~h}$ statin treatment; (b) HepG2 cellular cholesterol following $24 \mathrm{~h}$ statin treatment. ${ }^{* *} p<0.01,{ }^{* * *} p<0.001$, $^{* * * *} p<0.0001$ compared to control.

\subsection{The Effect of Statins on HDAC Activity in Cultured Cells}

No statin (at $10 \mu \mathrm{M})$ inhibited HDAC activity in live HepG2 cells after $24 \mathrm{~h}$ treatment (Figure 2a). Conversely, sodium butyrate and trichostatin A (TSA), both established HDAC inhibitors, significantly inhibited HDAC activity $(p<0.0001)$. Atorvastatin, chosen as a model statin due to its potency and widespread use, was tested at a range of concentrations and did not inhibit HDAC activity at up to and including $50 \mu \mathrm{M}$ (Figure S2), a concentration sufficiently high to impact cell viability, and more than sufficient to reduce cellular cholesterol content. Statins similarly failed to inhibit HDAC activity in MDA-MB-231 breast carcinoma cells and BRIN-BD11 insulin-secreting cells, while sodium butyrate and TSA showed significant inhibition $(p<0.0001)$ (Figure $2 b, c)$.

Since statins were reported to directly inhibit HDAC activity in A549 lung carcinoma nuclear extracts [12], we examined the direct effect of statins on HDAC activity in HepG2 nuclear extracts. As in live cells, atorvastatin and pravastatin did not inhibit HDAC activity at $100 \mu \mathrm{M}$ (Figure 2d), a dose significantly higher than the $10 \mu \mathrm{M}$ used by Lin et al. [12]. By contrast, and as in live cells, TSA strongly inhibited HDAC activity $(p<0.0001)$. A similar lack of effect was found with statins in MDA-MB-231 nuclear extracts (Figure S3).

Additionally, experiments were conducted using the Fluor De Lys ${ }^{\circledR}$ HDAC fluorometric activity assay kit, also used in previous studies by Lin et al. [12], Chen et al. [13], and Singh et al. [11]. HDAC inhibition was observed with TSA $(p<0.0001)$ and sodium butyrate $(p<0.0001)$ but not with statins in either HepG2 nuclear extracts or in the HeLa cervical adenocarcinoma nuclear extract provided with the kit (Figure 3).

\subsection{HDAC Activity in the Liver of Statin-Treated Mice}

We next utilized liver samples from a previous animal study by our group in which male C57B1/6J mice were fed normal chow or a high-fat diet and treated with atorvastatin for 12 weeks. HDAC activity was measured in nuclear extracts isolated from the liver of control animals fed a normal or high-fat diet and from atorvastatin-treated animals fed a normal or high-fat diet. As for studies on live cells 
and nuclear extracts, we found no effect with statin treatment on HDAC activity compared to the control (Figure 4). Furthermore, there was no effect of diet on HDAC activity.

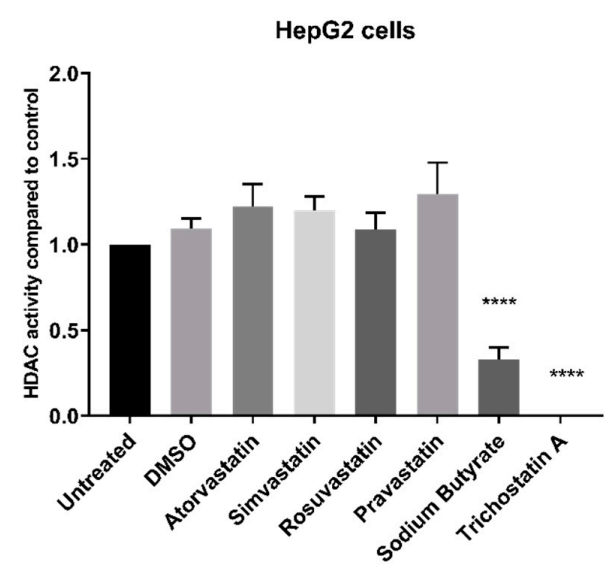

(a)

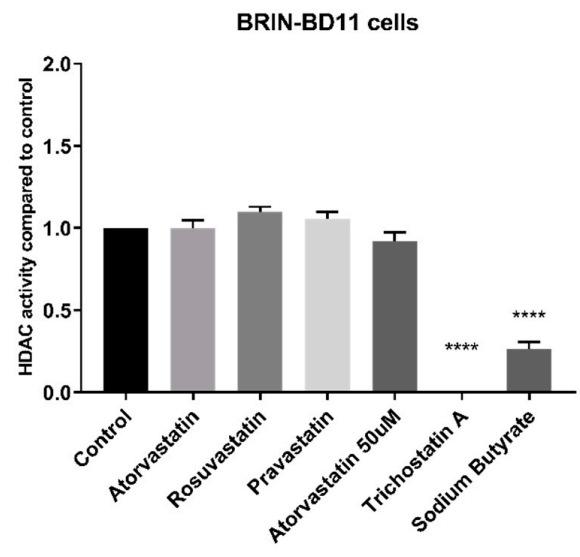

(c)

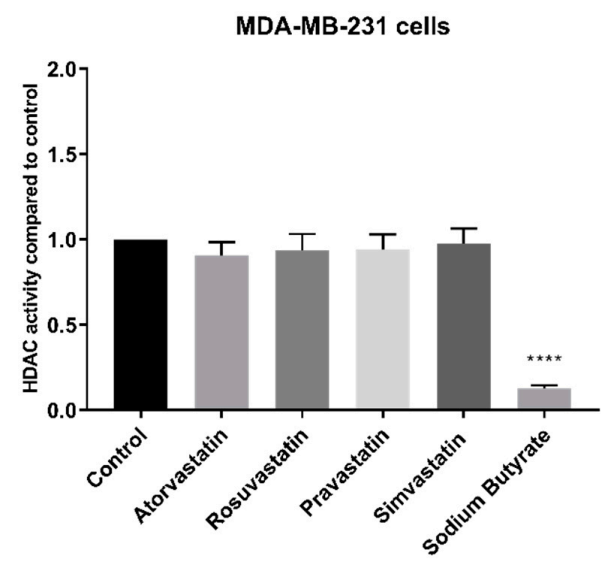

(b)

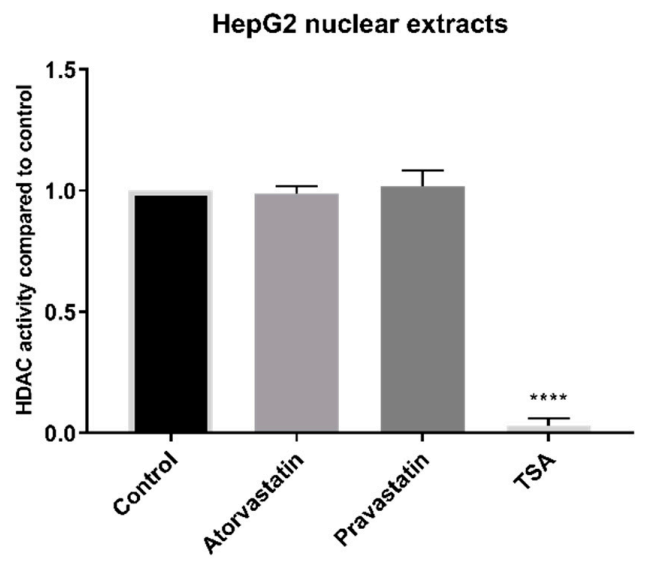

(d)

Figure 2. HDAC activity using the Sigma-Aldrich In Situ HDAC activity kit. (a) HDAC activity of live HepG2 cells following $24 \mathrm{~h}$ treatment with $10 \mu \mathrm{M}$ statins; (b) HDAC activity of live MDA-MB-231 cells following $24 \mathrm{~h}$ treatment with $10 \mu \mathrm{M}$ statins; (c) HDAC activity of live BRIN-BD11 cells following $24 \mathrm{~h}$ treatment with $10 \mu \mathrm{M}$ statins; and (d) HDAC activity following direct application of $100 \mu \mathrm{M}$ statins to HepG2 nuclear extracts. ${ }^{* * * *} p<0.0001$ compared to control.

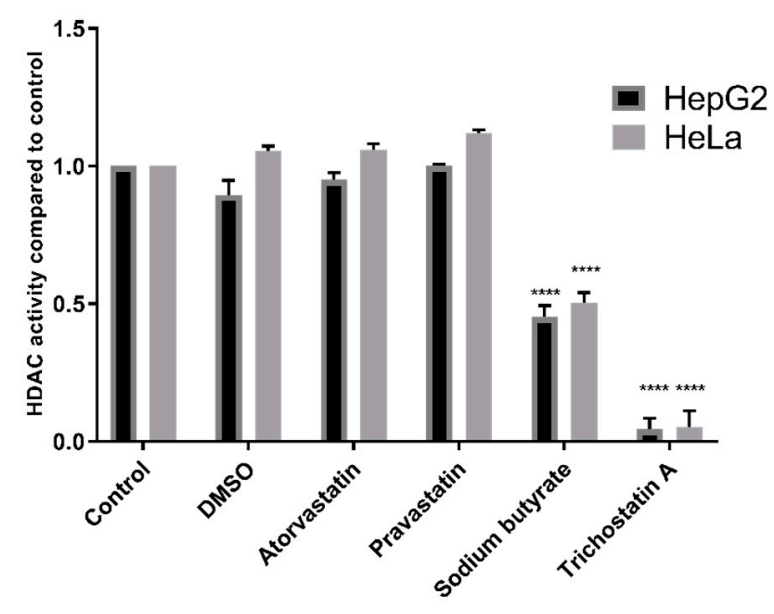

Figure 3. HDAC activity of HepG2 and HeLa nuclear extracts treated with $200 \mu \mathrm{M}$ statins, $1 \mathrm{mM}$ sodium butyrate, or $4 \mu \mathrm{M}$ TSA using the Fluor De Lys ${ }^{\circledR}$ HDAC activity kit. **** $p<0.0001$ compared to control. 


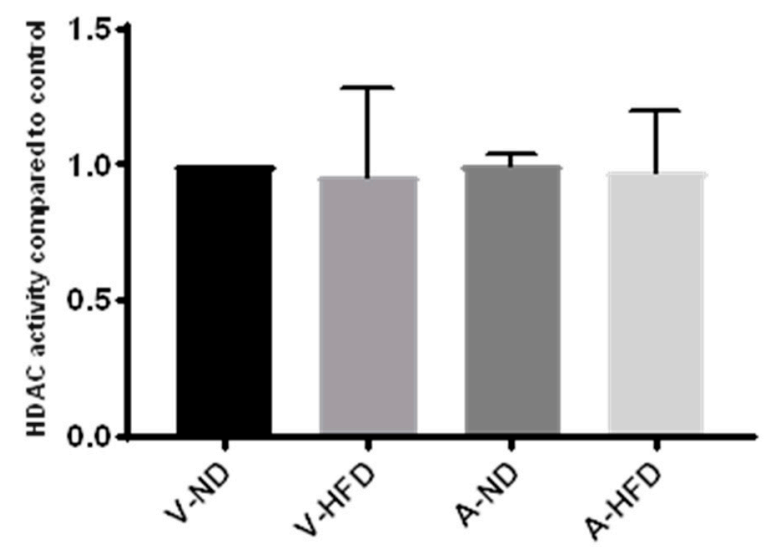

Figure 4. HDAC activity in nuclear extracts from livers of mice on a normal diet (ND) or high-fat diet (HFD) treated with atorvastatin (A) or water (V) for 12 weeks. $n=3$ per group.

\subsection{The Effect of Atorvastatin on HAT Activity in HepG2 Nuclear Extracts}

In addition to HDAC inhibition, compounds may alter histone acetylation levels through influences on HATs. However, a $100 \mu \mathrm{M}$ dose of atorvastatin had no effect on HAT activity when applied directly to HepG2 nuclear extracts (Figure 5). Conversely, and as expected, the control HAT inhibitor curcumin reduced the HAT activity of HepG2 nuclear extracts by $70 \%$ ( $\pm 6 \% \mathrm{SEM}$ ).

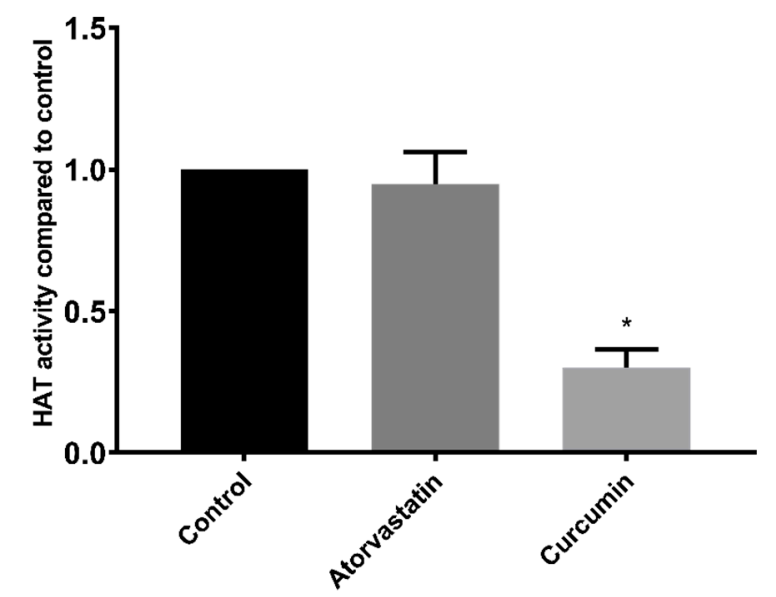

Figure 5. HAT activity of HepG2 nuclear extracts treated with $100 \mu \mathrm{M}$ atorvastatin or curcumin. * $p<0.05$ compared to control.

\subsection{The Effects of Atorvastatin Treatment on Global Histone Modifications}

Baseline levels of histone H3K9 and H4 acetylation were low in HepG2 cells (Figure 6a,b). As expected, acetylation was increased in a dose-dependent manner by sodium butyrate treatment. Conversely, acetylation levels remained at a low baseline level in cells treated with atorvastatin. Similar results were seen in BRIN-BD11 cells (Figure S4). Baseline levels of histone acetylation were higher in THP-1 leukemic macrophages and were further increased by $5 \mathrm{mM}$ sodium butyrate treatment, but atorvastatin had no effect (Figure 6c,d).

In order to ascertain if the lack of lipoproteins in the media affected results, experiments were also conducted in HepG2 cells treated in high-lipoprotein serum (HLPS). The results obtained in HLPS were almost identical to those in lipoprotein-deficient serum (LPDS), with sodium butyrate drastically increasing H3K9 acetylation, and atorvastatin having no effect (Figure S5). 

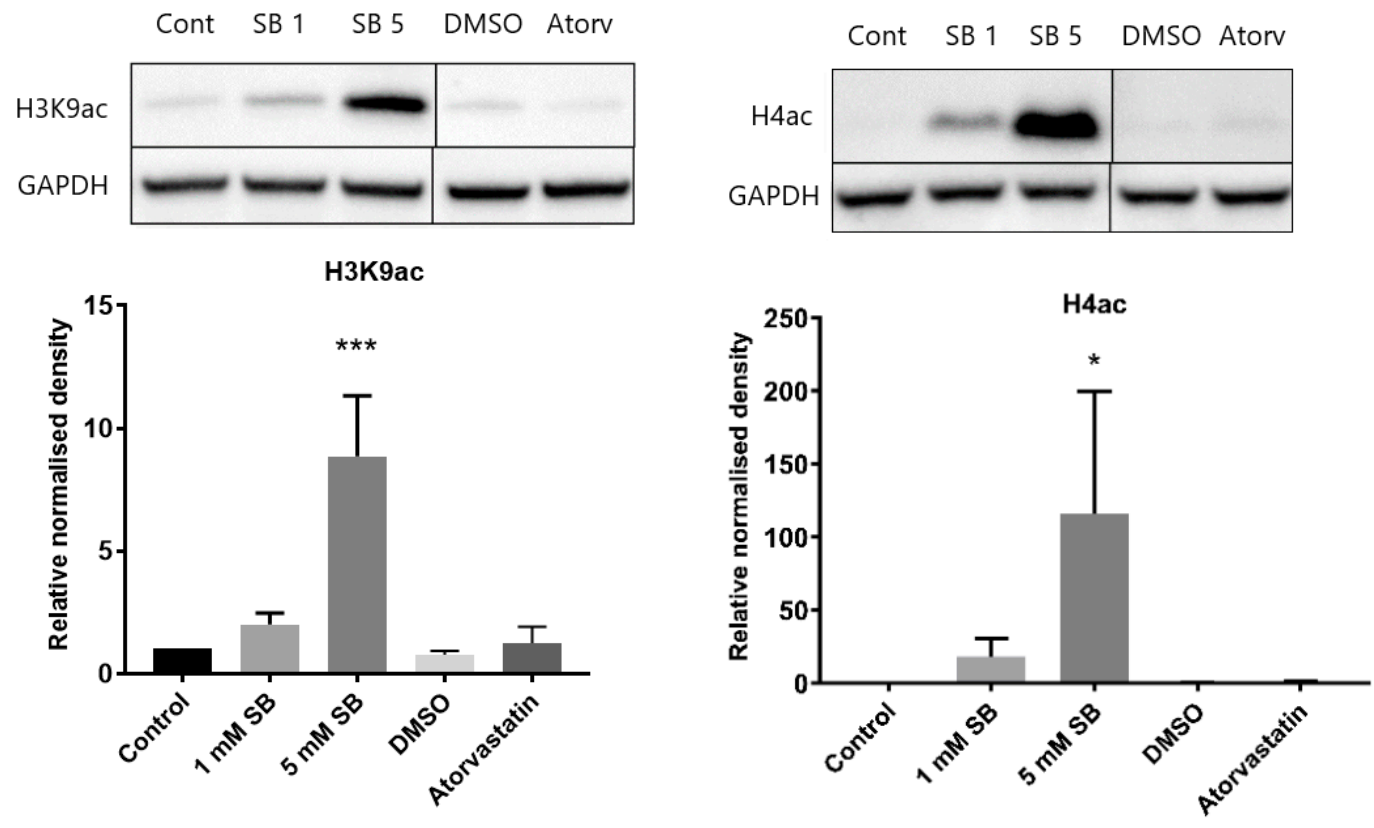

(a)
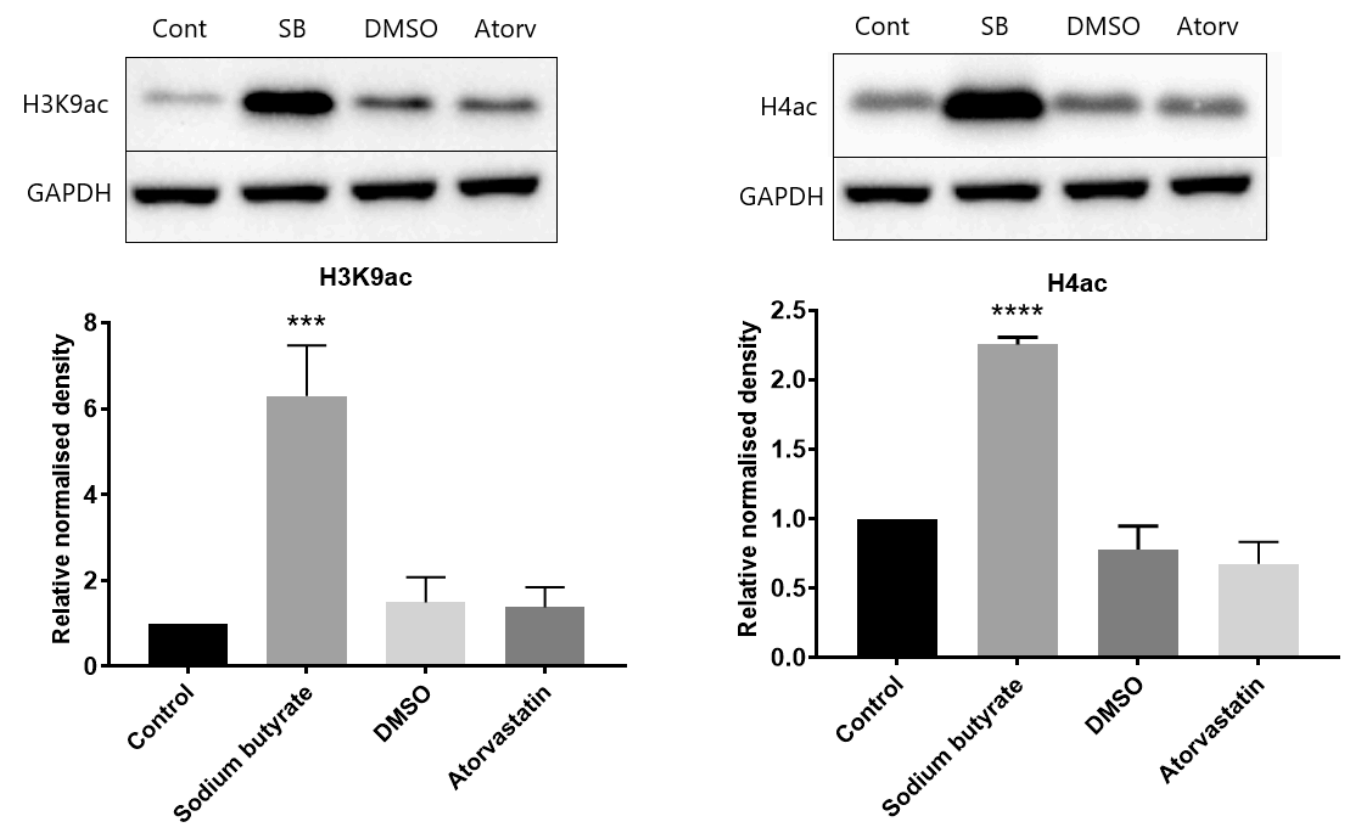

(c)

(d)

Figure 6. Influence of atorvastatin on histone acetylation in HepG2 and THP-1 cell extracts by Western blot analysis. (a) Relative level of acetylated histone H3K9 in HepG2 cells; (b) relative level of acetylated histone H4 in HepG2 cells; (c) relative level of acetylated histone H3K9 in THP-1 cells; and (d) relative level of acetylated histone H4 in THP-1 cells. Cells were treated for $24 \mathrm{~h}$ with $1 \mathrm{mM}$ or $5 \mathrm{mM}$ sodium butyrate (SB), $10 \mu \mathrm{M}$ atorvastatin, or equivalent DMSO vehicle control. The graphs illustrate combined density readings normalized to GAPDH from three independent experiments. ${ }^{*} p<0.05,{ }^{* * *} p<0.001$, **** $p<0.0001$.

\subsection{The Effect of Atorvastatin on DNMT Activity}

Statins have also been reported to inhibit DNMT activity [14]. When applied directly to HepG2 whole cell extracts, $200 \mu \mathrm{M}$ atorvastatin did not significantly inhibit, and in fact tended to increase, DNMT activity (Figure 7a). Conversely, curcumin, a known DNMT inhibitor, decreased DNMT activity by $65 \%$ ( $\pm 8 \%$ SEM). This was significantly greater than equal dose of the rationally designed DNMT 
inhibitor RG108, which inhibited DNMT activity by 25\% ( $\pm 9 \%$ SEM) and was just above the threshold for significance $(p=0.057)$. Similar results were obtained with BRIN-BD11 cell extracts (Figure S6).

DNMT activity was also measured in the extracts of cells that had been treated for $24 \mathrm{~h}$ with atorvastatin or curcumin. DNMT activity was quite variable in these experiments but DNMT activity of cells treated with atorvastatin was in fact higher than the DMSO vehicle control $(p<0.01)$ (Figure 7b). In extracts of curcumin treated cells, DNMT activity was not significantly different than that of the control, possibly due to the rapid degradation of curcumin in media [18].

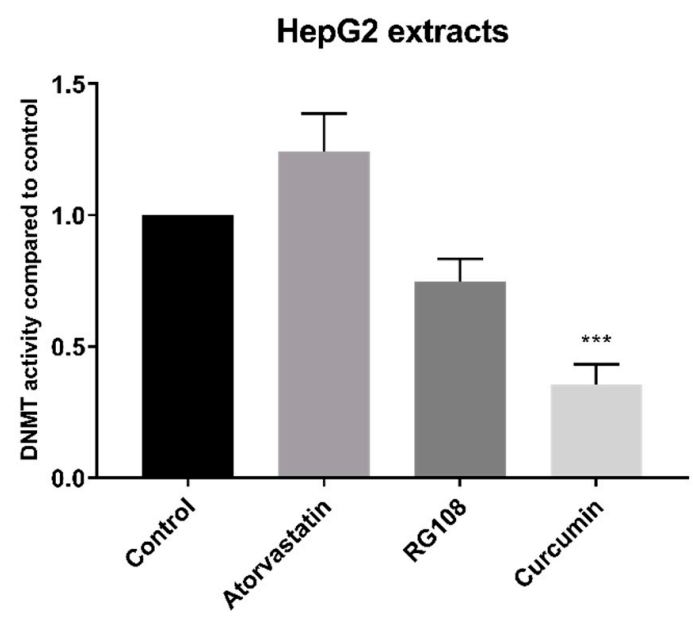

(a)

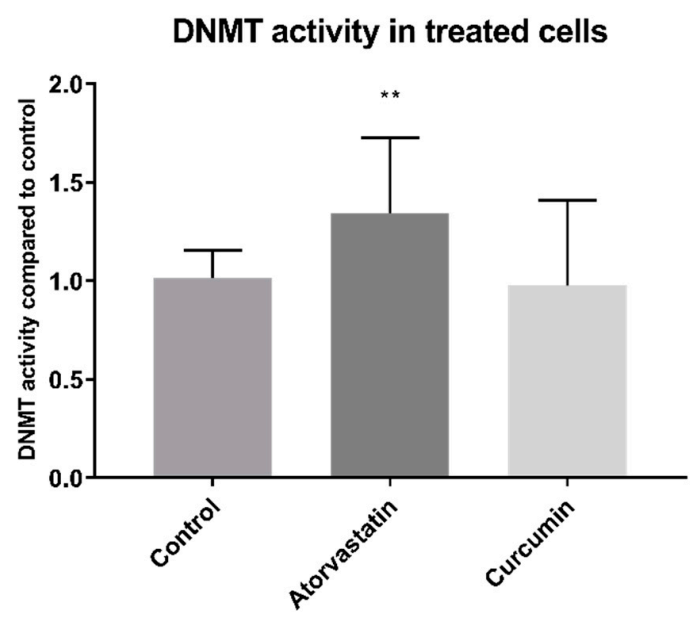

(b)

Figure 7. DNMT activity in HepG2 whole cell extracts. (a) DNMT activity in whole cell extracts treated directly with $200 \mu \mathrm{M}$ atorvastatin, RG-108 or curcumin. (b) DNMT activity in whole cell extracts from cells treated for $24 \mathrm{~h}$ with $10 \mu \mathrm{M}$ atorvastatin or curcumin. ${ }^{* *} p<0.01,{ }^{* * *} p<0.001$

\section{Discussion}

In view of their widespread use and tolerability, the potential anticancer effects of statins held considerable appeal. In vitro studies on cancer cell lines have revealed potential mechanisms for anticancer activity, including cell cycle arrest, induction of apoptosis and prevention of metastasis [19]. It had also been suggested that inhibition of epigenetic modifying enzymes, such as HDACs and DNMTs, may contribute to the anticancer activity of statins, as we reviewed previously [7]. Epigenetic modifications are often altered in cancer cells and upregulation of HDACs and DNMTs have been associated with numerous cancers [10]. As a result, multiple epigenetic modifying drugs have been approved for cancer treatment, including the HDAC inhibitors vorinostat, romidepsin, belinostat, panobinostat, and chidamide, and the DNMT inhibitors azacytidine and decitabine [20]. However, these are associated with severe side effects and treatment-related deaths have been reported in clinical trials [21]. Thus, based on previous studies, statins offered potential benefits over current epigenetic drugs for cancer. However, our results indicate that direct inhibition of major epigenetic enzymes is unlikely to contribute to any anticancer effects of statins.

Studies of various cancer cell lines [12,22], a macrophage line [23], and a rabbit model of atherosclerosis [24] reported that statin treatment can cause hyperacetylation of histones $\mathrm{H} 3$ and $\mathrm{H} 4$, either globally or in certain genomic regions, or inhibition of HDAC activity and expression. Our extensive results on HDAC activity in live cells are in complete contrast to these previous studies. This was demonstrated on a variety of different cells lines, namely HepG2, MDA-MB-231, and BRIN-BD11 cells, in addition to livers of mice treated with statins for 12 weeks, leading us to conclude that neither short nor long term statin treatment alters cellular HDAC activity. We therefore considered the possibility that in live cells the statins may be unable to enter the nucleus, or that some other element of cellular activity may prevent them from interacting with HDAC proteins, as the studies by other groups were conducted either using recombinant HDAC proteins [13] or nuclear 
extracts [11,12]. To address this possibility, we conducted experiments using nuclear extracts, and yet again failed to find any HDAC inhibition by statins. The negative outcome of these experiments is unlikely to be due to inappropriately low statin concentration, as concentrations of up to $200 \mu \mathrm{M}$ statins were used in the nuclear extract experiments. This concentration is significantly higher than the $10 \mu \mathrm{M}$ doses reported to inhibit HDAC activity by Lin et al. [12] and well above the 1-15 nM mean serum concentration of statin users [25]. Furthermore, measurement of cellular cholesterol demonstrated that the statins did lower cellular cholesterol, indicating that the statins successfully entered the cells and inhibited HMG-CoA reductase. The contrast in results could also be due to the different cell types or the use of different HDAC substrates; previous studies used A549 lung carcinoma cells and primary glomerular mesangial cells, and all reported studies used the Fluor-De-Lys ${ }^{\circledR}$ HDAC Activity Assay Kit. Concerning the latter, we also utilized the Fluor-De-Lys ${ }^{\circledR}$ assay kit and found no effect with statins on HDAC activity in HepG2 or HeLa nuclear extracts. Concerning the former, we did not use the exact cell types previously used in the literature; however, we used a wider range of cell types than previous studies, and also examined liver tissue extracts; all cell lines responded as expected to established HDAC inhibitors.

It could be argued that focusing on other specific cell or cancer types may have been a better approach, but in those circumstances, failure to find an effect would not preclude an effect in other cell types. Our initial studies were conducted in one cell type, namely HepG2 cells; once those studies were found to be negative, we took the approach of examining for effects in a range of cell types. We considered this to be appropriate on the basis of the mechanisms involved for agents with well-established effects such as butyrate and curcumin which mediate their effects by direct interaction with epigenetic enzymes and which are thus efficacious in most cell types. We reason that by using a variety of different cell types, we have shown there is a general lack of effect of statins on the enzymes examined. Furthermore, by conducting experiments using nuclear extracts we can deduce that statins do not inhibit activity through direct interaction with the HDAC enzyme, as posited by Lin et al. [12], while experiments with live cells and animal tissue suggest that statins do not alter HDAC activity indirectly through phenotypic or metabolic changes, although we concede that this cannot be ruled out in all circumstances.

In contrast to studies on HDACs, the effect of statins on HAT activity has not been previously reported. However, as with HDAC activity, our results were unable to demonstrate a direct effect of atorvastatin on HAT activity in HepG2 nuclear extracts.

There are some limitations to the use of fluorogenic HDAC substrates in activity assays. In particular, the Fluor-de-Lys ${ }^{\mathrm{TM}}$ substrate has been shown to interfere with HDAC activity and tends to overestimate inhibition of HDAC1 and HDAC6, while failing to detect inhibition of HDAC8 [26]. Similar experiments have not been reported for the cell permeable substrate used in the Sigma-Aldrich assay kit which we also used in our studies. However, the lack of HDAC and HAT inhibition in these assays is supported by the immunoassay results showing no increase in acetylation of histone H3K9 or multiple residues of histone H4. In fact, while Chen et al. [13] reported inhibition of recombinant HDAC1, HDAC2, and HDAC6 proteins by lovastatin and atorvastatin, they did not find an increase in histone $\mathrm{H} 3$ acetylation following $24 \mathrm{~h}$ treatment of A549 cells with 30-50 $\mu \mathrm{M}$ lovastatin. Conversely, Lin et al. [12] did report increased H3 acetylation following $16 \mathrm{~h}$ of treatment in A549 cells with $30 \mu \mathrm{M}$ statins (lovastatin, simvastatin, pravastatin, fluvastatin, and atorvastatin).

Our results also showed that DNMT activity was not decreased by $24 \mathrm{~h}$ prior treatment with atorvastatin. By contrast, the opposite was found with an increase in DNMT activity. There is a caveat, since there a lack of an appropriate control inhibitor for this assay for studies on live cells and in the context of a control for a prolonged $24 \mathrm{~h}$ exposure, as explained later. However, our finding of no decreases in DNMT activity by statins in live cells is supported by a similar finding in cell extract experiments, where statins were added directly to cell extracts, and where we had a well-established DNMT inhibitor control, namely curcumin [27]. This enables us to conclude that statins do not directly inhibit DNMT activity. That $24 \mathrm{~h}$ curcumin treatment of cells did not reduce DNMT activity in live 
cells, has been observed elsewhere and is possibly due to degradation of curcumin in cell media [18]. Indeed the most well-established DNMT inhibitor-5-azacytidine (as well as other established DNMT inhibitors decitabine and zebularine)—does not interact with free DNMTs, instead inhibiting DNMT activity through its incorporation into DNA, and hence does not show inhibition in DNMT activity assays [28]. For this reason, combined with the marginal nonsignificance $(p=0.057)$ of the rationally designed inhibitor RG108 on cell extracts, we consider that there is a lack of appropriate established DNMT inhibitors to use in cell culture, which remains a challenge to be addressed in all such studies.

Our DNMT findings are in contrast with the results of Kodach et al. [14], who reported reduced DNMT activity in nuclear extracts from lovastatin treated HCT116 colon cancer cells. Several differences in design of our study and the latter study may account for the disparate results. Firstly, they used lovastatin, whereas we chose to use atorvastatin as our exemplar statin given the latter is the most commonly prescribed statin in numerous advanced economies including the United States [29], England [30], and Australia [31], while lovastatin is not available for therapeutic use in some regions. Additionally, Kodach et al. [14] used a different cell type and a longer treatment time, $48 \mathrm{~h}$ versus $24 \mathrm{~h}$. Since these authors did not investigate if lovastatin directly inhibited DNMT enzyme activity, it cannot be ruled out that the observed decrease in activity is a result of changed phenotype, as they did observe increased differentiation and reduced 'stemness' of the cancer cells. Finally, different commercial kits were used, although both relied on similar principles based on use of an unmethylated DNA substrate and Adomet (S-adenosyl methionine) as a methyl donor, followed by measurement of methylation of the DNA of using a methylation specific antibody reporter system.

\section{Materials and Methods}

\subsection{Preparation of Stock Solutions of Treatments}

Atorvastatin calcium salt, rosuvastatin calcium salt, simvastatin sodium salt and pravastatin sodium salt (Cayman Chemical, Ann Arbor, MI, USA), curcumin, and RG108 (Abcam, Cambridge, UK) were dissolved in dimethyl sulfoxide (DMSO) (Sigma-Aldrich, St. Louis, MO, USA) to a concentration of $10 \mathrm{mM}$ and stored at $-20{ }^{\circ} \mathrm{C}$ for short-term use or at $-80{ }^{\circ} \mathrm{C}$ for long-term use. Sodium butyrate (Selleck Chemicals, Houston, TX, USA) was dissolved in ultrapure distilled water to a concentration of $50 \mathrm{mM}$ and stored at $-20^{\circ} \mathrm{C}$.

\subsection{Cell Culture and Treatments}

HepG2 human hepatocellular carcinoma cells were obtained from ATCC and provided by Dr Ross Graham (Curtin University, Perth, Australia). MDA-MB-231 human breast carcinoma cells were obtained from ATCC and provided by Professor Arunasalam Dharmarajan (Curtin University, Bentley, WA, Australia). BRIN-BD11 rat insulinoma cells were from Cell Bank Australia, deposited by Prof PR Flatt, University of Ulster, via the European Tissue Culture Collection. THP-1 human leukemic monocytes were obtained from Dr Hilary Warren (Canberra Hospital, Canberra, Australia) and provided via Professor Deirdre Coombe's team (Curtin University, Bentley, WA, Australia). HepG2 cells were maintained in Dulbecco's Modified Eagle's medium: Ham's F-12 Nutrient Mixture (DMEM: F12 media) (Sigma-Aldrich) supplemented with 10\% fetal bovine serum (FBS) (Serana, Bunbury, WA, Australia). MDA-MB-231 cells, BRIN-BD11 cells, and THP-1 cells were maintained in Roswell Park Memorial Institute (RPMI) media (Sigma-Aldrich) supplemented with 10\% FBS. All cells were maintained in $25-\mathrm{cm}^{2}$ or $75-\mathrm{cm}^{2}$ tissue culture flasks at $37^{\circ} \mathrm{C}$ in a humidified incubator equilibrated with $5 \% \mathrm{CO}_{2}$. All cells tested negative for mycoplasma contamination. Cells were seeded in 96-well plates or $25-\mathrm{cm}^{2}$ or $75-\mathrm{cm}^{2}$ tissue culture flasks and allowed to recover prior to treatment. Prior to use, THP- 1 cells were differentiated into macrophages by the addition of $50 \mathrm{nM}$ phorbol 12-myristate 13-acetate (PMA) (Sigma-Aldrich) to the culture media. THP-1 cells were differentiated for $48 \mathrm{~h}$, then allowed to recover for 1 day before treatment. All cells were treated in media supplemented 
with 10\% lipoprotein-deficient serum (LPDS) unless otherwise stated. Control cells were treated with DMSO as a vehicle control unless otherwise stated.

\subsection{Preparation of Lipoprotein-Deficient Serum and High-Lipoprotein Serum}

The density of FBS was adjusted to $1.21 \mathrm{~g} / \mathrm{mL}$ with sodium bromide. The solution was transferred to $11.5 \mathrm{~mL}$ polyallomer ultracrimp tubes and centrifuged for $20 \mathrm{~h}$ at $70,000 \times \mathrm{g}$ in a Sorvall $\mathrm{WX}$ ultracentrifuge. The base of the tube was pierced with a butterfly needle and $1 \mathrm{~mL}$ aliquots collected. The cholesterol and total protein of the aliquots was measured using the Amplex ${ }^{\mathrm{TM}}$ Red Cholesterol Assay Kit (Life Technologies, Carlsbad, CA, USA) and the Pierce BCA Protein Assay Kit (ThermoFisher, Waltham, MA, USA), respectively, to determine which aliquots were deficient in lipoproteins (LPDS) and those enriched in lipoproteins (HLPS). Salt was removed from the serum by centrifugation with Zeba $^{\mathrm{TM}}$ desalting spin columns $7 \mathrm{~K}$ MWCO (ThermoFisher) and serum was sterilized with a Millex 0.22 um syringe filter prior to storage at $-20{ }^{\circ} \mathrm{C}$.

\subsection{Animal Studies}

Eight-week-old male C57B1/6J mice from the Animal Resource Centre, Murdoch, Western Australia were delivered to the animal facility, Curtin University. Following acclimatization, mice were randomly assigned to either normal diet (ND) or high-fat diet (HFD) (Specialty Feeds, Glen Forrest, WA, Australia). From week 4, mice were further divided into treatment groups to receive either $10 \mathrm{mg} / \mathrm{kg}$ / day of atorvastatin or vehicle (water) by gastric gavage for a further 12 weeks. Following completion of the treatment period, mice were starved for $6 \mathrm{~h}$ and anesthetized in an isoflurane chamber before being euthanized by cervical dislocation. Liver samples were removed from the carcass and collected in prechilled microcentrifuge tubes and snap-frozen either in dry ice or liquid nitrogen. Animal experiments were approved by Curtin University's Animal Ethics Committee (AEC_2016_17, approval date 14 April 2016).

\subsection{Nuclear Extraction}

Nuclear extraction was conducted using the Abcam Nuclear Extraction Kit according to the manufacturer's instructions. For experiments on cultured cells, confluent cells were scraped and incubated on ice for $10 \mathrm{~min}$ in pre-extraction buffer containing dithiothreitol (DTT) and protease inhibitor cocktail (PIC), then centrifuged at 12,000 rpm for $1 \mathrm{~min}$. The cytoplasmic supernatant was removed, and the pellets incubated on ice for $15 \mathrm{~min}$ in nuclear extraction buffer containing DTT and PIC, then sonicated 3 times for $10 \mathrm{~s}$. The extract was then centrifuged at $14,000 \mathrm{rpm}$ for $10 \mathrm{~min}$ at $4{ }^{\circ} \mathrm{C}$ and the supernatant removed for protein quantification using the Pierce Coomassie Protein Assay Kit (ThermoFisher). For experiments on murine liver, samples were placed in a glass homogenizer containing pre-extraction buffer with DTT and PIC and homogenized manually. Homogenized samples were transferred to microcentrifuge tubes and incubated on ice for $15 \mathrm{~min}$ then centrifuged at $10,000 \mathrm{rpm}$ for $10 \mathrm{~min}$ at $4{ }^{\circ} \mathrm{C}$. The cytoplasmic supernatant was removed, and nuclear extraction continued as for cultured cells.

\subsection{Whole Cell Protein Extraction}

Whole cell extractions were only conducted on cultured cells. Confluent cells were scraped then lysed on ice for $20 \mathrm{~min}$ in radioimmunoprecipitation assay (RIPA) lysis buffer (Sigma-Aldrich) containing protease and phosphatase inhibitors (Sigma-Aldrich). The lysate was sonicated 10 times for $10 \mathrm{~s}$ then centrifuged at 14,000 rpm for $10 \mathrm{~min}$ and the protein rich supernatant was removed for protein quantification using the Pierce BCA Protein Assay Kit (ThermoFisher). 


\subsection{Viability Assays}

Viability was determined using alamarBlue ${ }^{\circledR}$ (ThermoFisher) as a measure of total cellular metabolism. Following $22 \mathrm{~h}$ treatment, $10 \mu \mathrm{L}$ of alamarBlue ${ }^{\circledR}$ was added to the treatment media and cells were incubated for a further two hours. Fluorescence was then read by the EnSpire Multimode Plate Reader (PerkinElmer, Waltham, MA, USA) with an excitation wavelength of $540 \mathrm{~nm}$ and an emission wavelength of $590 \mathrm{~nm}$.

\subsection{Cellular Cholesterol Content}

After $24 \mathrm{~h}$ of statin treatment, the culture medium was removed and lipids were extracted using 3:2 hexane:isopropanol. Lipids were transferred to a $96 \mathrm{~V}$ well plate and allowed to dry at room temperature. Cholesterol content was assessed using the Amplex Red Cholesterol Assay Kit (Life Technologies). Samples were dissolved in a reaction buffer and isopropanol mixture (1:1) and incubated with catalase for $15 \mathrm{~min}$ at $37^{\circ} \mathrm{C}$ to eliminate endogenous peroxides which would otherwise interfere in this assay [32]. Amplex Red working solution was added and following 30 min incubation, fluorescence was read with an excitation wavelength of $540 \mathrm{~nm}$ and an emission wavelength of $590 \mathrm{~nm}$.

\subsection{HDAC Activity}

The In Situ Histone Deacetylase (HDAC) Activity Fluorometric Assay Kit (Sigma-Aldrich) was used to measure HDAC activity according to the manufacturer's instructions. The HDAC substrate was added to wells containing live cells directly to the treatment media after $23 \mathrm{~h}$ treatment. For HDAC activity assays on the nuclear extracts, enzyme substrate was added concurrently with statin treatments in phosphate-buffered saline. After 1-h incubation, the HDAC developer was added and the plate was incubated for a further $30 \mathrm{~min}$ and fluorescence read with an excitation wavelength of $368 \mathrm{~nm}$ and an emission wavelength of $442 \mathrm{~nm}$.

Confirmatory experiments were carried out with the Fluor De Lys ${ }^{\circledR}$ HDAC fluorometric activity assay kit (Enzo Life Sciences, Farmingdale, NY, USA) according to the manufacturer's instructions. HeLa nuclear extract provided with the kit and HepG2 nuclear extract prepared as above were incubated with treatments and substrate for $30 \mathrm{~min}$. Following incubation with developer for $10 \mathrm{~min}$, fluorescence was read with an excitation wavelength of $360 \mathrm{~nm}$ and an emission wavelength of $460 \mathrm{~nm}$.

\subsection{HAT Activity Assays}

HAT activity was measured with the EpiQuik ${ }^{\mathrm{TM}}$ HAT Activity/Inhibition Assay Kit (Epigentek, Farmingdale, NY, USA) according to the manufacturer's instructions. The HAT substrate was captured in strip wells for $45 \mathrm{~min}$. Following washing, strip wells were incubated with acetyl-CoA, HepG2 nuclear extracts, and treatments for $90 \mathrm{~min}$ at $37^{\circ} \mathrm{C}$. Following washing, wells were incubated sequentially with the capture antibody and detection antibody, followed by the addition of the developer solution. Once the solution in the control wells changed to a medium blue, the enzymatic reaction was stopped with the stop solution and the absorbance read at $450 \mathrm{~nm}$.

\subsection{DNMT Activity Assays}

DNMT activity was measured with the Abcam DNMT Activity Assay Kit according to the manufacturer's instructions, with the following modification; whole cell extracts were used as opposed to nuclear extracts since experiments with nuclear extracts were not successful. For direct DNMT inhibition experiments cell extracts were incubated with $200 \mu \mathrm{M}$ atorvastatin, curcumin or RG-108 and the Adomet methyl donor at $37^{\circ} \mathrm{C}$ for $2 \mathrm{~h}$ in strip wells coated with DNMT substrate. For treated cells, equal amounts of total protein were incubated with Adomet. Following incubation and washing, wells were incubated sequentially with the capture antibody, detection antibody, and enhancer solution, followed by the addition of the developer solution. Once the solution in the control wells changed to a 
medium blue, the enzymatic reaction was stopped with the stop solution and the absorbance read at $450 \mathrm{~nm}$ with a reference wavelength of $655 \mathrm{~nm}$.

\subsection{Immunoblotting for Modified Histones}

Equal amounts of protein were fractionated on Bolt ${ }^{\mathrm{TM}} 4-12 \%$ Bis-Tris Plus Gels (ThermoFisher) following denaturing at $98{ }^{\circ} \mathrm{C}$ for $10 \mathrm{~min}$. Proteins were transferred onto nitrocellulose membranes using the iBlot Gel Transfer Device (Invitrogen, Carlsbad, CA, USA) and blocked with 3\% bovine serum albumin (Bovogen, Keilor East, VIC, Australia) in Tris-buffered saline with $0.1 \%$ Tween 20 (TBST) for at least $1 \mathrm{~h}$. Membranes were incubated overnight with primary antibodies against acetylated H3K9 and acetylated H4 (S1, K5, K8, and K12) (Santa Cruz, Dallas, TX, USA) diluted in blocking buffer. GAPDH (Abcam) was used as a housekeeping reference protein. Membranes were incubated with goat anti-mouse IgG H\&L horseradish peroxidase (HRP) (Abcam) in blocking buffer for at least one hour, then washed 3 times with TBST. Immunodetection was performed with the Amersham ECL Prime Western Blotting Detection Reagent (GE Healthcare, Chicago, IL, USA) in the Chemi-Doc ${ }^{\text {TM }}$ Gel Imaging System (Bio-Rad, Hercules, CA, USA). Band density was measured with Image Lab 6.0.1 software (Bio-Rad) and normalized to the density of housekeeping protein GAPDH or total protein as determined by Coomassie Blue staining.

\subsection{Statistical Analysis}

At least three independent experiments were conducted. Statistical significance was determined with analysis of variance (ANOVA) with results considered significant if $p<0.05$ using GraphPad Prism 8.0.1 software (GraphPad Software, San Diego, CA, USA).

\section{Conclusions}

There is substantial interest in inhibitors of epigenetic enzymes as anticancer agents: that commonly prescribed drugs, such as statins, may confer anticancer activities via epigenetic mechanisms is therefore an attractive proposition. However, our results indicate that statins are unlikely to inhibit the three major classes of epigenetic modifying enzymes, namely HDACs, HATs, and DNMTs, and in fact may increase DNMT activity. Our comprehensive studies of HDAC activity in multiple cell types, in both live cells and nuclear extracts and using two different HDAC substrates demonstrate no effect with statin treatment. While our in vitro studies indicate it is unlikely that statins directly inhibit these epigenetic enzymes, it is feasible that through effects on cell metabolism and/or phenotype, statins may have an indirect effect as described previously for metformin, particularly with prolonged treatment. However, in the context of HDACs, our studies on the livers of statin treated mice would also seem to rule out effects of prolonged treatment on HDAC activity, direct or otherwise. It cannot be ruled out that statins may influence subclasses of these various enzymes or the recruitment of enzymes to certain genomic regions without altering the overall activity. Thus, statins may influence epigenetic modifications in the proximity of certain genes and thereby affect gene expression through epigenetic means. To that end, targeted studies of specific genomic regions are warranted and are currently under investigation.

Supplementary Materials: The following are available online at http:/ / www.mdpi.com/2072-6694/11/4/516/s1, Figure S1: The effect of $24 \mathrm{~h}$ statin treatment on the viability of MDA-MB-231 cells, Figure S2: HDAC activity of HepG2 cells following $24 \mathrm{~h}$ atorvastatin treatment, Figure S3: HDAC activity of MDA-MB-231 nuclear extracts, Figure S4: Immunoblotting of BRIN-BD11 cell extracts, Figure S5: Immunoblotting of extracts of HepG2 cells treated in HLPS, Figure S6: DNMT activity of BRIN-BD11 cell extracts.

Author Contributions: Conceptualization, S.B. and C.D.S.M.; Methodology, S.B., G.E., T.S., and C.D.S.M.; Software, S.B. and W.N.; Validation, S.B.; Formal Analysis, S.B. and W.N.; Investigation, S.B., W.N., G.E., and T.S., Resources, P.N. and C.D.S.M.; Data Curation, S.B. and W.N.; Writing-Original Draft Preparation, S.B.; Writing-Review and Editing, S.B., G.E., T.S., P.E.M., P.N., and C.D.S.M.; Visualization, S.B.; Supervision, P.E.M., P.N., and C.M; Project Administration, C.D.S.M.; Funding Acquisition, S.B. and C.D.S.M. 
Funding: This research was supported by an Australian Government Research Training Program Scholarship and by the School of Pharmacy and Biomedical Sciences, Curtin University.

Acknowledgments: We wish to acknowledge the facilities and technical support provided by the Curtin Health Innovation Research Institute.

Conflicts of Interest: The authors declare no conflict of interest.

\section{References}

1. Johansen, M.E.; Green, L.A.; Sen, A.; Kircher, S.; Richardson, C.R. Cardiovascular risk and statin use in the United States. Ann. Fam. Med. 2014, 12, 215-223. [CrossRef]

2. Naci, H.; Brugts, J.J.; Fleurence, R.; Tsoi, B.; Toor, H.; Ades, A.E. Comparative benefits of statins in the primary and secondary prevention of major coronary events and all-cause mortality: A network meta-analysis of placebo-controlled and active-comparator trials. Eur. J. Prev. Cardiol. 2013, 20, 641-657. [CrossRef]

3. Kavalipati, N.; Shah, J.; Ramakrishan, A.; Vasnawala, H. Pleiotropic effects of statins. Indian J. Endocrinol. Metab. 2015, 19, 554-562. [CrossRef]

4. Undela, K.; Shah, C.S.; Mothe, R.K. Statin use and risk of cancer: An overview of meta-analyses. World J. Meta-Anal. 2017, 5, 41-53. [CrossRef]

5. Kim, M.; Myung, S.; Tran, B.; Park, B. Statins and risk of cancer: A meta-analysis of randomized, double-blind, placebo-controlled trials. Indian J. Cancer 2017, 54, 470-477. [CrossRef]

6. Mei, Z.; Liang, M.; Li, L.; Zhang, Y.; Wang, Q.; Yang, W. Effects of statins on cancer mortality and progression: A systematic review and meta-analysis of 95 cohorts including 1,111,407 individuals. Int. J. Cancer 2017, 140, 1068-1081. [CrossRef] [PubMed]

7. Allen, S.C.; Mamotte, C.D.S. Pleiotropic and adverse effects of statins-Do epigenetics play a role? J. Pharmacol. Exp. Ther. 2017, 362, 319-326. [CrossRef] [PubMed]

8. Ordovas, J.M.; Smith, C.E. Epigenetics and cardiovascular disease. Nat. Rev. Cardiol. 2010, 7, $510-519$. [CrossRef] [PubMed]

9. Burgio, E.; Lopomo, A.; Migliore, L. Obesity and diabetes: From genetics to epigenetics. Mol. Biol. Rep. 2015, 42, 799-818. [CrossRef]

10. Kanwal, R.; Gupta, S. Epigenetic modifications in cancer. Clin. Genet. 2012, 81, 303-311. [CrossRef]

11. Singh, R.S.; Chaudhary, D.K.; Mohan, A.; Kumar, P.; Chaturvedi, C.P.; Ecelbarger, C.M.; Godbole, M.M.; Tiwari, S. Greater efficacy of atorvastatin versus a non-statin lipid-lowering agent against renal injury: Potential role as a histone deacetylase inhibitor. Sci. Rep. 2016, 6, 38034. [CrossRef]

12. Lin, Y.C.; Lin, J.H.; Chou, C.W.; Chang, Y.F.; Yeh, S.H.; Chen, C.C. Statins increase p21 through inhibition of histone deacetylase activity and release of promoter-associated HDAC1/2. Cancer Res. 2008, 68, 2375-2383. [CrossRef]

13. Chen, J.-B.; Chern, T.-R.; Wei, T.-T.; Chen, C.-C.; Lin, J.-H.; Fang, J.-M. Design and synthesis of dual-action inhibitors targeting histone deacetylases and 3-hydroxy-3-methylglutaryl coenzyme A reductase for cancer treatment. J. Med. Chem. 2013, 56, 3645-3655. [CrossRef]

14. Kodach, L.L.; Jacobs, R.J.; Voorneveld, P.W.; Wildenberg, M.E.; Verspaget, H.W.; van Wezel, T.; Morreau, H.; Hommes, D.W.; Peppelenbosch, M.P.; van den Brink, G.R.; et al. Statins augment the chemosensitivity of colorectal cancer cells inducing epigenetic reprogramming and reducing colorectal cancer cell 'stemness' via the bone morphogenetic protein pathway. Gut 2011, 60, 1544-1553. [CrossRef]

15. Aiman, U.; Najmi, A.; Khan, R.A. Statin induced diabetes and its clinical implications. J. Pharmacol. Pharmacother. 2014, 5, 181-185. [CrossRef]

16. Codd, R.; Braich, N.; Liu, J.; Soe, C.Z.; Pakchung, A.A.H. Zn(II)-dependent histone deacetylase inhibitors: Suberoylanilide hydroxamic acid and trichostatin A. Int. J. Biochem. Cell Biol. 2009, 41, 736-739. [CrossRef]

17. Bridgeman, S.C.; Ellison, G.C.; Melton, P.E.; Newsholme, P.; Mamotte, C.D.S. Epigenetic effects of metformin: From molecular mechanisms to clinical implications. Diabetes Obes. Metab. 2018, 20, 1553-1562. [CrossRef]

18. Wang, Y.J.; Pan, M.H.; Cheng, A.L.; Lin, L.I.; Ho, Y.S.; Hsieh, C.Y.; Lin, J.K. Stability of curcumin in buffer solutions and characterization of its degradation products. J. Pharm. Biomed. Anal. 1997, 15, 1867-1876. [CrossRef]

19. Zaleska, M.; Mozenska, O.; Bil, J. Statins use and cancer: An update. Future Oncol. 2018, 14, 1497-1509. [CrossRef] 
20. Liu, Z.; Gao, Y.; Li, X. Cancer epigenetics and the potential of epigenetic drugs for treating solid tumors. Expert Rev. Anticancer Ther. 2019, 19, 139-149. [CrossRef]

21. Mottamal, M.; Zheng, S.; Huang, T.L.; Wang, G. Histone deacetylase inhibitors in clinical studies as templates for new anticancer agents. Molecules 2015, 20, 3898-3941. [CrossRef]

22. Karlic, H.; Thaler, R.; Gerner, C.; Grunt, T.; Proestling, K.; Haider, F.; Varga, F. Inhibition of the mevalonate pathway affects epigenetic regulation in cancer cells. Cancer Genet. 2015, 208, 241-252. [CrossRef]

23. Feig, J.E.; Shang, Y.; Rotllan, N.; Vengrenyuk, Y.; Wu, C.; Shamir, R.; Torra, I.P.; Fernandez-Hernando, C.; Fisher, E.A.; Garabedian, M.J. Statins promote the regression of atherosclerosis via activation of the CCR7-dependent emigration pathway in macrophages. PLoS ONE 2011, 6, e28534. [CrossRef]

24. Tikoo, K.; Patel, G.; Kumar, S.; Karpe, P.A.; Sanghavi, M.; Malek, V.; Srinivasan, K. Tissue specific up regulation of ACE2 in rabbit model of atherosclerosis by atorvastatin: Role of epigenetic histone modifications. Biochem. Pharmacol. 2015, 93, 343-351. [CrossRef]

25. Björkhem-Bergman, L.; Lindh, J.D.; Bergman, P. What is a relevant statin concentration in cell experiments claiming pleiotropic effects? Br. J. Clin. Pharmacol. 2011, 72, 164-165. [CrossRef]

26. Sodji, Q.H.; Kornacki, J.R.; Mrksich, M.; Oyelere, A.K. In Vitro Histone Deacetylase Activity Screening: Making a Case for Better Assays. In Epigenetic Technological Applications; Zheng, Y.G., Ed.; Academic Press: Boston, MA, USA, 2015; pp. 319-332.

27. Liu, Z.; Xie, Z.; Jones, W.; Pavlovicz, R.E.; Liu, S.; Yu, J.; Li, P.-K.; Lin, J.; Fuchs, J.R.; Marcucci, G.; et al. Curcumin is a potent DNA hypomethylation agent. Bioorg. Med. Chem. Lett. 2009, 19, 706-709. [CrossRef]

28. Brueckner, B.; Garcia Boy, R.; Siedlecki, P.; Musch, T.; Kliem, H.C.; Zielenkiewicz, P.; Suhai, S.; Wiessler, M.; Lyko, F. Epigenetic reactivation of tumor suppressor genes by a novel small-molecule inhibitor of human DNA methyltransferases. Cancer Res. 2005, 65, 6305-6311. [CrossRef]

29. Medical Expenditure Panel Survey. Prescribed Drug Estimates: 2015. Available online: https://meps.ahrq. gov / data_stats/summ_tables/hc/drugs/2015/hcdrugest_totpur2015.shtml (accessed on 13 February 2019).

30. Prescribing and Medicine Team. Prescriptions Dispensed in the Community—Statistics for England, 2007-2017; NHS Digital; Available online: https://digital.nhs.uk/data-and-information/publications/statistical/ prescriptions-dispensed-in-the-community / prescriptions-dispensed-in-the-community-england---2007--2017 (accessed on 13 February 2019).

31. PBS Information Management Section. Expenditure and Prescriptions Twelve Months to 30 June 2018; Australian Government, 2018. Available online: http://www.pbs.gov.au/statistics/expenditureprescriptions / 2017-2018/expenditure-and-prescriptions-twelve-months-to-30-june-2018.pdf (accessed on 13 February 2019).

32. Jové, M.; Serrano, J.C.E.; Bellmunt, M.J.; Cassanyé, A.; Anglès, N.; Reguant, J.; Morelló, J.R.; Pamplona, R.; Portero-Otín, M. When cholesterol is not cholesterol: A note on the enzymatic determination of its concentration in model systems containing vegetable extracts. Lipids Health Dis. 2010, 9, 65. [CrossRef] 